\title{
MODELO DE EMPRENDIMIENTO PARA LA FACULTAD DE CIENCIAS ADMINISTRATIVAS Y ECONÓMICAS DE LA UNIVERSIDAD FRANCISCO DE PAULA SANTANDER OCAÑA
}

\section{ENTREPRENEURSHIP MODEL FOR THE FACULTY OF MANAGEMENT AND ECONOMICS AT THE FRANCISCO DE PAULA SANTANDER OCAÑA UNIVERSITY}

\author{
MSc. Isbelia Karina Rincón Parada ${ }^{\mathrm{a}}$, Esp. Karina Yulieth Casadiego Suarez ${ }^{\mathrm{b}}$, \\ MSc.Marta Milena Peñaranda Peñaranda ${ }^{c}$
}

${ }^{\text {a }}$ Universidad Francisco de Paula Santander Ocaña, Grupo de Investigación GRUCITE, Vía Acolsure Sede el Algodonal, Ocaña - Norte de Santander, Colombia, ikrinconp@ufpso.edu.co

${ }^{\mathrm{b}}$ Universidad Francisco de Paula Santander Ocaña, Grupo de Investigación GIDSE, Vía Acolsure Sede el Algodonal, Ocaña - Norte de Santander, Colombia

${ }^{c}$ Universidad Francisco de Paula Santander Ocaña, Grupo de Investigación GIDSE, Vía Acolsure Sede el Algodonal, Ocaña-Norte de Santander, Colombia, mmpenarandap@ufpso.edu.co

\begin{abstract}
Resumen: Las Universidades hoy en día deben ser emprendedoras, llevando el conocimiento académico a un contexto empresarial. En la medida que las Universidades contribuyan en el desarrollo socioeconómico de las naciones, se genera una nueva cadena de valor para la creación de nuevas empresas. El artículo presenta el modelo de emprendimiento para la Facultad de Ciencias Administrativas y Económicas de la Universidad Francisco de Paula Santander Ocaña, el cual comprende dos elementos intrínsecamente relacionados, la innovación y el emprendimiento, para que los estudiantes, grupos de investigación, egresados y emprendedores, puedan desarrollar con éxito la formulación y la puesta en marcha de una empresa.

En este sentido, el tipo de investigación utilizada fue exploratoria; por lo tanto no se definió una hipótesis y en cuanto al el diseño se utilizó un estudio documental, en donde la información recolectada aportó nuevos conocimientos a partir de datos secundarios que proporcionó la Universidad Nacional de Colombia Sede Manizales y otras IES que tienen definido un modelo de emprendimiento.
\end{abstract}


Palabras Claves: Emprendimiento, Innovación, Empresarios, Incubación, Responsabilidad Socio Empresarial.

\begin{abstract}
Abastract: The Universities today must be entrepreneurial, bringing the academic knowledge to a business context. To the extent that universities contribute to the socioeconomic development of nations, a new value chain for the creation of new companies is generated. The article presents the model of entrepreneurship for the Faculty of Administrative and Economic Sciences of the Francisco de Paula Santander Ocaña University, which comprises two elements intrinsically related, innovation and entrepreneurship for students, research groups, alumni and entrepreneurs can successfully develop the formulation and implementation of a company.
\end{abstract}

In this sense, the type of research used was exploratory; therefore a hypothesis is not defined and as to design a documentary study, where the information collected provided new knowledge from secondary data provided by the Nacional de Colombia University sectional Manizales and other higher education institutions that have defined a model was used entrepreneurship.

Keywords: Entrepreneurship, Innovation, Business, Incubation, Corporate Social Responsibility.

\section{Introducción}

En un ambiente caracterizado por el constante cambio de las organizaciones, las universidades no están al margen de estos procesos y se han venido transformando a través de la historia. A finales del siglo XIX y principios del siglo $\mathrm{XX}$ se presentó en el ámbito universitario, lo que los expertos han denominado la "primera revolución académica" (Morales, 2008), caracterizada por la generación del conocimiento como segunda misión de las universidades.

Esta segunda revolución académica, plantea la tercera misión para las Universidades "Contribuir al desarrollo socioeconómico de las naciones" Molas-
Gallart, Salter, Patel, Scotty Duran (2002) plantean que la tercera misión está relacionada "con la generación, uso, aplicación y explotación del conocimiento y otras capacidades de la Universidad fuera del contexto académico". El nuevo modelo de universidad que responde a estas expectativas ha sido denominado la "Universidad emprendedora", la cual involucra las tres misiones de una universidad (Smilor, Dietrich y Gibson, 1993; Etzkowitz, 1998).

Este modelo de Universidad Emprendedora, comprende dos elementos intrínsecamente relacionados: la Innovación y el Emprendimiento. La acción de 
emprender puede tener y de hecho la tiene, una marcada connotación social por su naturaleza creadora de riqueza, en todas sus manifestaciones. Se debe tener como como objetivo impulsar la Cultura Emprendedora, a través del desarrollo de un Sistema de Emprendimiento que contribuya al desarrollo socio-económico, fortaleciendo los vínculos universidad-empresa-estado que haga más pertinente la misión de la Universidad Francisco de Paula Ocaña.

El gobierno nacional evidenciando la necesidad de dar soporte y acompañamiento en las nuevas empresas durante sus primeros años tanto de gestión como de operación, crea las incubadoras de empresas como instituciones dedicadas a facilitar el desarrollo de nuevas unidades productivas mediante gestión en la búsqueda de recursos financieros, la generación de contactos comerciales y la asesoría integral en la estructuración y operación de un buen plan de negocios (Cruz Gartner \& Matiz Bulla, 2004). Por esta razón, se desarrolla el Sistema Nacional de Creación e Incubación de Empresas - Colombia (SNCIE) liderado por el Servicio Nacional de Aprendizaje (SENA), el cual tiene como objetivo articular los esfuerzos nacionales para el desarrollo de la cadena de valor para la creación de nuevas empresas.

En este sentido el Ministerio de Comercio, Industria y Turismo (2009) desarrolla las políticas de emprendimiento, las cuales en su primer rol “(...) (i) promover alianzas públicas- privadasacadémicas" incluye a la academia como fomento del emprendimiento y la incubación de empresas bajo un enfoque basado en el conocimiento. Es así como en el decreto 1192 de-2009, se reglamentan las Redes Regionales de Emprendimiento.

Cabe resaltar que universidades como la Nacional de Colombia, la UIS (la primera vez que se escribe una sigla, se debe escribir el nombre completo), la ICESI, la pontificia Bolivariana, la Salle, la Cooperativa de Colombia, entre otras, han venido desarrollando desde la academia procesos de emprendimiento en sus estudiantes, aportando al desarrollo social y económico nacional, local y regional.

En este mismo orden y dirección, el acuerdo No. 082 de 2011 de la Universidad Francisco de Paula Santander adopta las políticas de extensión y proyección social en 
su Artículo Segundo numeral j “(...) Es compromiso de la Universidad, fomentar el progreso de la región, incentivando el espíritu emprendedor de sus estudiantes, formándolos en emprendimiento $y$ acompañándolos en sus proyectos de generación de empresa". Por lo anterior, este proyecto prende crear el modelo de emprendimiento para la Facultad de Ciencias administrativas y Económicas de la Universidad Francisco de Paula Santander Ocaña, el cual servirá de manera transversal para todas las facultades de la institución, que tengan como compromiso el emprendimiento en sus estudiantes y egresados.

Teniendo en cuenta todo lo anterior, se formuló la siguiente pregunta de investigación: ¿El modelo de emprendimiento fortalecerá el compromiso de la Universidad en incentivar el espíritu emprendedor de los estudiantes y fomentará la cultura de emprendimiento en los procesos y proyectos de generación de empresa?, la cual se le dio respuesta mediante los siguientes objetivos específicos: Identificar las diferentes etapas del modelo de emprendimiento para la Facultad de Ciencias Administrativas y
Económicas, articular la estrategia Plan Formador de Competencias Empresarial, en la conformación del modelo de emprendimiento para la Facultad de Ciencias Administrativas y Económicas e implementar el modelo de emprendimiento en el Centro de Proyección Empresarial, adscrita a la Facultad de Ciencias Administrativas y Económicas.

\section{Metodología}

El diseño del modelo de emprendimiento para la Facultad de Ciencias Administrativas y Económicas de la Universidad Francisco de Paula Santander Ocaña, comprende dos elementos intrínsecamente relacionados: la innovación y el emprendimiento. El cual permitirá que los estudiantes, grupos de investigación, egresados y emprendedores, puedan desarrollar con éxito la formulación y la puesta en marcha/operación de una empresa.

Para tal fin, el enfoque de la investigación fue cualitativo y su profundidad exploratoria, debido a que no se puede llegar a una conclusión sobre qué etapas son relevantes y cuáles no, en un modelo de emprendimiento. 
El diseño para la recolección de los datos fue documental, a partir de la información secundaria que nos proporcionó la Universidad Nacional de Colombia Sede Manizales y otras IES que tienen definido un modelo de emprendimiento. El propósito de la invitación era aplicarse en el primer Centro de Proyección Empresarial impulsado por la Universidad Francisco de Paula Santander Ocaña.

Las población que se tomó como referencia para el estudio, fueron los funcionarios de los Centros de Emprendimiento de la IES en Colombia y los emprendedores que se encuentran en su etapa de incubación.

El método de muestreo utilizado fue no probabilístico por conveniencia, en donde se tomaron al azar algunas Universidades públicas que crearon un modelo de emprendimiento propio y lo han implementado en sus Centros de Emprendimiento. En este caso, la Universidad de Nacional sede Manizales, Universidad de los Andes, Universidad
Javeriana, EAFIT, Universidad de Antioquia y la Universidad del Norte.

La técnica de recolección de información utilizada fue la observación documental, en la cual se extrajeron datos de interés de las universidades que tienen un modelo de emprendimiento y que su implantación ha tenido casos de éxito en la formulación y creación de empresas por parte de estudiantes y egresados.

Cabe señalar, que como es una investigación exploratoria, se utilizó la entrevista no estructurada focalizada individual, cuyo objetivo era identificar si los modelos de emprendimientos, han dado como resultado la creación de empresas y que estas a su vez hayan aportado a la generación de empleo.

\section{Resultados}

Las fases que utilizaron para dar respuesta a la pregunta de investigación y los resultados parciales obtenidos son:

Fase 1. Identificación de las diferentes etapas del modelo de emprendimiento para 
la Facultad de Ciencias Administrativas y Económicas: Se observó el modelo de emprendimiento de la Universidad Nacional sede Manizales, presentó ocho etapas administradas por el Parque Nacional de Innovación Empresarial y el Consultorio Administrativo, como se puede observar en la figura 1. Una particularidad de este modelo, es su transversalidad con todos los programas académicos que dicta la Institución, así como el soporte que se le da a la comunidad que no hace parte de la Universidad.

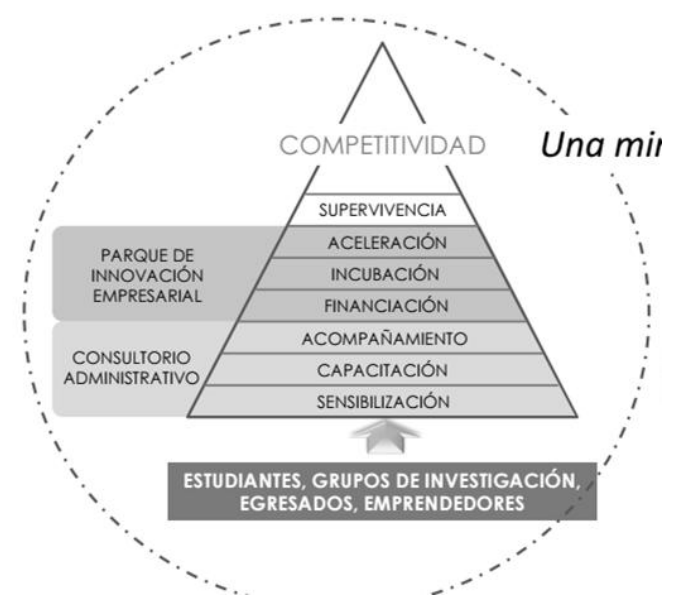

Figura 1. Modelo de Emprendimiento Universidad Nacional Sede Manizales (datos suministrados por los participantes.

Fuente: Elaboración propia.

Adicionalmente al modelo de emprendimiento, han desarrollado su propia herramienta para identificar modelos de negocio innovadores, como se evidencia en la figura 2. El primer paso de la herramienta consiste en identificar el modelo de negocio en la organización, por medio de una matriz compuesta por un grupo de preguntas que abarcan nueve bloques temáticos. El siguiente paso es la valoración del modelo de negocio mediante una matriz que fusiona el análisis DOFA junto con el Business Model Canvas, en donde se evalúan los aspectos internos de la empresa (fortalezas y debilidades) y posteriormente la posición de la empresa con el entorno (oportunidades y amenazas). Finalmente, con una matriz comparativa y mediante la abstracción gráfica de un árbol, se pueden identificar los factores determinantes para que ese modelo de negocios pueda llegar a ser innovador.

Con los datos obtenidos, se realizó el primer bosquejo del modelo de emprendimiento para la UFPSO de acuerdo con el contexto de la institución y de la Facultad de Ciencias Administrativas y Económicas. El modelo consta de siete eslabones como se observa en la figura 3 , cuya finalidad es desarrollar los procesos de fomento, promoción, proyección empresarial y apoyo al emprendimiento; articulándolos con los programas académicos, prestando servicios de asesoría, consultoría a los estudiantes, 
grupos de investigación, egresados y

emprendedores de los diversos sectores

sociales y empresariales de la región.

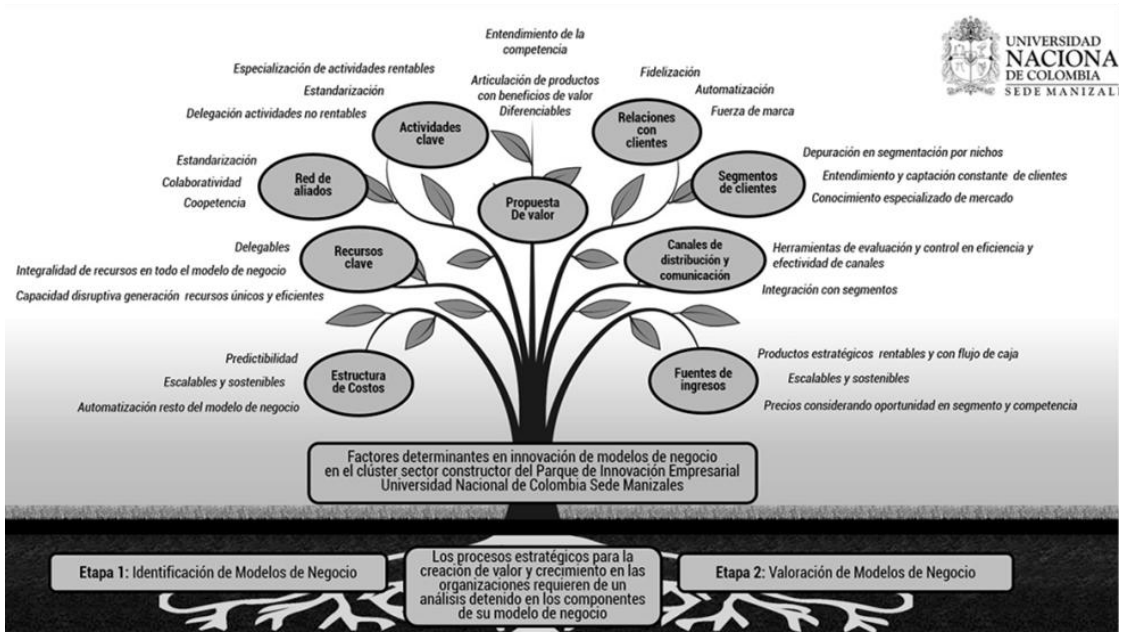

Figura 2 Herramienta para identificar modelos de negocios innovadores, en el parque de innovación empresarial (datos suministrados por los participantes).

Fuente: Elaboración propia.

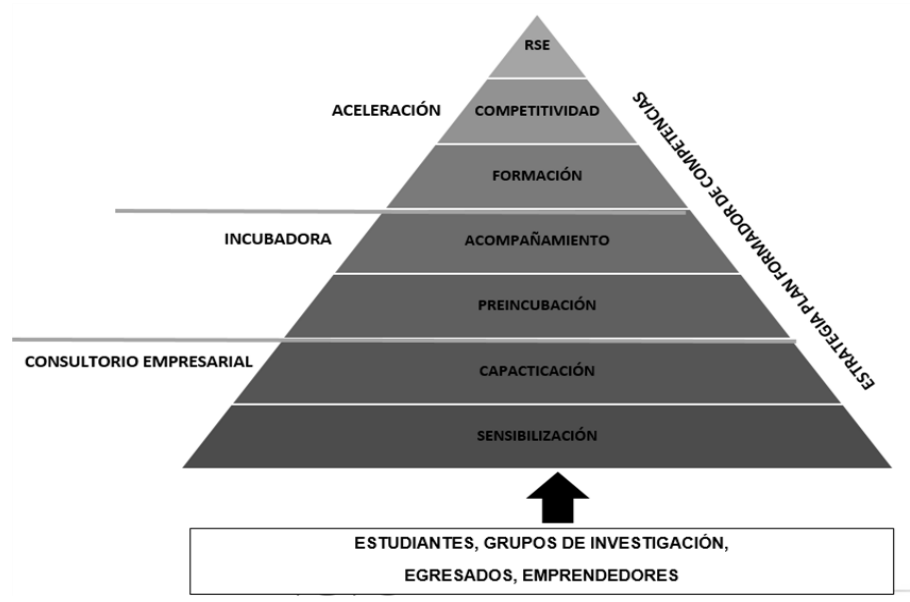

Figura 3. Modelo de emprendimiento de la UFPSO, versión No.1 (datos recabados por los autores).

Fuente: Elaboración propia.

Lo que se pretendió con el modelo es:

Primero, que el consultorio Empresarial tuviese inmerso dentro de sus funciones las actividades de sensibilización y capacitación, que estarían a cargo de los consultorios administrativos y el consultorio contable y tributario.

El objetivo del consultorio es prestar servicios de información, asesoría y capacitación a estudiantes, grupos de investigación, egresados y emprendedores de empresas de los diferentes sectores de la economía para el fortalecimiento del emprendimiento y la innovación.

Segundo, el proceso de incubación desarrollaría el acompañamiento y apoyo para la estructuración de las ideas 
de negocio identificadas en los estudiantes, egresados y sectores productivos. Por lo que su misión sería estimular y fomentar la cultura de emprendimiento e innovación, acompañando la estructuración de las iniciativas empresariales, su creación, operación y sostenibilidad.

Tercero, el proceso de aceleración convertirá las nuevas empresas en unidades altamente competitivas maximizando el valor agregado de la compañía a través de la internacionalización y la innovación continua que les permita repensar el negocio constantemente. Así mismo, generar en los empresarios una responsabilidad social empresarial, lo que indica que deberá potencializar los emprendimientos para que generen valor y logren en el corto plazo el crecimiento, la innovación y la escalabilidad que garantice su éxito empresarial.

Fase 2. Articulación de la estrategia Plan Formador de Competencias Empresariales (PFCE), en la conformación del modelo de emprendimiento para la Facultad de Ciencias Administrativas y Económicas: Una vez se tenga definida el modelo de emprendimiento final, se articulará la estrategia plan formador de competencias (EPFCE) para que los estudiantes del programa de Administración de Empresas sean el insumo que fortalezcan el modelo de emprendimiento. Así mismo, este PFCE, se pueda enseñar a los grupos de investigación, egresados y emprendedores de los diversos sectores sociales $y$ empresariales de la región.

Lo anterior con el fin, que cuando se implemente el modelo no se presenten diferencias o desventajas entre los emprendedores externos a la Facultad de Ciencias Administrativas y Económicas.

Fase 3. Implementación del modelo de emprendimiento en el Centro de Proyección Empresarial, adscrita a la Facultad de Ciencias Administrativas y Económicas: Una vez se tenga el modelo emprendimiento diseñado, este será el modelo a seguir por el Centro de Proyección empresarial. El cual tiene como misión desarrollar los procesos de fomento, promoción, proyección empresarial y apoyo al emprendimiento, articulándolos con los programas académicos, prestando servicios de asesoría, consultoría a los estudiantes, grupos de investigación, egresados y emprendedores de 
los diversos sectores sociales $y$

empresariales de la región.

\section{Bibliografía}

Congreso de la República de Colombia (2006). Ley 1014 de 2006 por la cual se expide la ley De fomento a la cultura del emprendimiento.

Cruz Gartner. A. C., \& Matiz Bulla, F. J. M. (2004). Sistema nacional de creación e incubación de empresas.

$\begin{array}{crr}\text { Ministerio de } & \text { Industria y } & \text { Comercio } \\ \text { Republica } & \text { de } & \text { Colombia } \\ \text { (2009).Política de Emprendimiento }\end{array}$
Molas-Gallart, J., P. Tang, S. Morrow, T. Sinclair and B. Martin. 1999. Assessing Research Impact on NonAcademic Audiences Final Report to the Economic and Social Research Council. SPRU, Brighton.

Morales Gualdrón, S. T. (2008). El emprendedor académico y la decisión de crear Spin off: Un análisis del caso español.

Smilor, Raymond W., David V. Gibson and Glenn B. Dietrich, 1990;Spin-out Companies: Technology Start-ups from UT-Austin', Journal of Business Venturing 